\section{Cambia el color y el riesgo... Nuevo escenario para el cáncer de próstata en Chile}

\author{
ELIANA ANDAHUR ${ }^{1, \mathrm{a}}$, ALEJANDRO MERCADO ${ }^{1}$, \\ CATHERINE SÁNCHEZ ${ }^{1, b}$
}

\section{Prostate cancer in african american immigrants in Chile}

In 2017, migrants were $4.35 \%$ of the Chilean population, mainly from Peru and Colombia. From 2015, the amount of migrants from Central America, particularly from Haiti increased notably. This process changed the phenotype of the male population, increasing the proportion of black men, mainly between 20 and 50 years. Afro-descendant men have a higher risk for prostate cancer, and the tumor can appear as early as 40 years of age among them. This increase will have future repercussions on the public health system, since part of these men have low income and poor living conditions. Therefore, it is necessary to discuss early detection strategies focused on this population, including education for both patients and health professionals. This review includes data on the reality of migration in Chile and its impact on the health system. The higher incidence and mortality of prostate cancer in the migrant population is reviewed and risk-adjusted screening strategies are proposed.

(Rev Med Chile 2019; 147: 1159-1166)

Key words: African Americans; Disparities; Health Status Disparities; Human Migration; Prostatic Neoplasms.

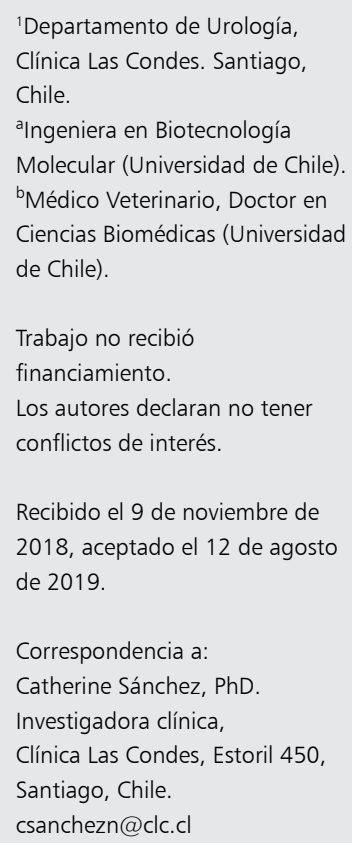

\section{La realidad de la inmigración en Chile.} Datos oficiales

$\mathrm{D}$ urante la historia de nuestro país, han ocurrido diversos procesos migratorios de importancia. Fenómenos políticos y sociales favorecieron la llegada de inmigrantes ingleses, italianos, árabes, judíos y alemanes, quienes se incorporaron a la sociedad chilena. A principio de los años noventa comenzó un nuevo proceso migratorio importante, gatillado por el crecimiento económico y la imagen de estabilidad política proyectada al exterior. Las crisis económicas en Argentina y Brasil posicionaron a Chile como una alternativa para los migrantes sudamericanos y en el último tiempo, del Caribe ${ }^{1}$.

Esto ha aumentado significativamente el número de inmigrantes en nuestro país, desde 114.597 personas en 1992 a 784.685 personas (4,35\% de la población) de acuerdo a datos del censo 2017 (Tabla 1)2. Este cambio se observó principalmente en la última década (Figura 1, izquierda), con una marcada tendencia al alza en el número de extranjeros, que se intensificó a partir del año 2015 (Figura 1, derecha) ${ }^{2}$.

La mayor cantidad de ingresos durante las últimas décadas ha sido de peruanos. Aunque, según datos del Departamento de Extranjería de 2016, durante la última década ha aumentado sostenidamente el ingreso de colombianos y bolivianos, mientras que la población de venezolanos, dominicanos y haitianos ha experimentado un reciente y explosivo incremento ${ }^{1}$. Según el censo de 2017, los principales países de procedencia son: Perú 
Tabla 1. Migrantes internacionales por residencia y lugar de nacimiento en distintos censos

\begin{tabular}{|c|c|c|c|}
\hline \multirow[t]{2}{*}{ Tipo de residencia } & \multicolumn{3}{|c|}{ Censos } \\
\hline & 1992 & 2002 & 2017 \\
\hline Total de nacidos en el extranjero & 114.597 & 197.929 & 784.685 \\
\hline Residentes habituales & 105.070 & 187.008 & 746.465 \\
\hline Población residente habitual con declaración de país de nacimiento & 13.041 .098 & 14.763 .250 & 17.150 .383 \\
\hline \% respecto de la población residente habitual con declaración de país de nacimiento & 0,81 & 1,27 & 4,35 \\
\hline
\end{tabular}

Fuente: Instituto Nacional de Estadísticas, Chile².

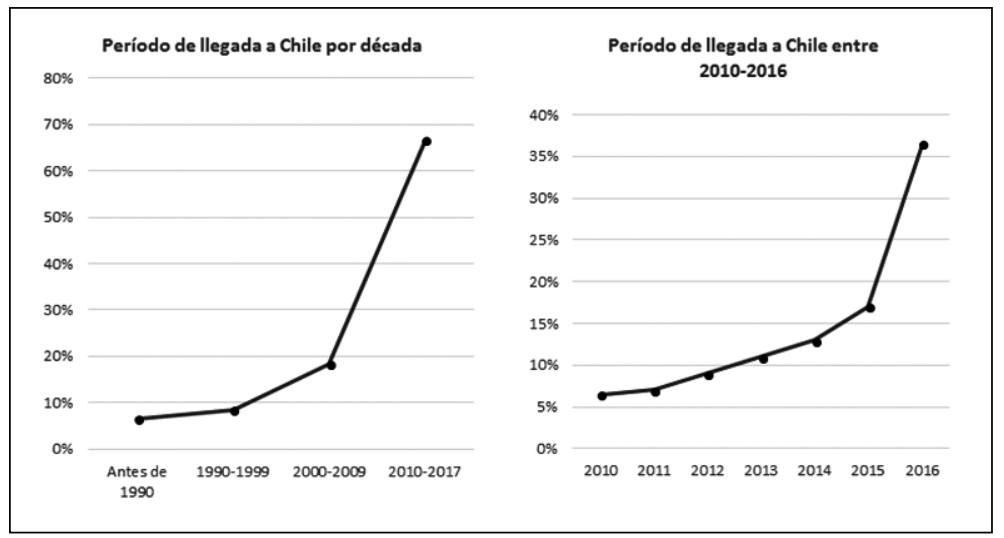

Figura 1. Porcentaje de inmigrantes internacionales según período de llegada al país, por década (izquierda) y del $66,7 \%$ de la última década (derecha) según el censo de 2017. Fuente: Instituto Nacional de Estadísticas, Chile².
(24,5\%), Colombia (13,8\%), Venezuela (10,9\%), Bolivia (9,9\%), Argentina (9,4\%), Haití $(8,2 \%)$ y Ecuador $(3,6 \%)$. Además, se debe considerar la población migrante ilegal, que ha incrementado y se estima entre 250 a 300 mil personas (según Policía de Investigaciones de Chile).

En la población inmigrante se observa una distribución similar respecto del número de hombres y mujeres (Figura 2). Sin embargo, es interesante notar que, en el caso de la población haitiana, hay una mayor proporción de hombres que llega y permanece en nuestro país. La consideración de la composición masculinizada de esta población es importante al analizar intervenciones a distinto nivel $^{3}$.

La migración también ha influido en el fenotipo de la población masculina chilena. Hace pocos años era difícil encontrar hombres de raza negra, sin embargo, en la actualidad ha aumentado la población afrodescendiente, proveniente principalmente de Colombia, Venezuela, Haití y Ecuador. A pesar de esto, en la actualidad no hay cifras oficiales de la población afrodescendiente, y sólo se discute sobre su inclusión laboral, educacional y cultural. En esta revisión, la discusión en cuanto a la llegada de hombres afrodescendientes a Chile se encuentra ajena de la discriminación racial, y enfocándose principalmente en los factores de riesgo de cáncer de próstata que presenta este grupo, lo que representa un nuevo escenario para la medicina nacional.

Por último, es necesario considerar los rangos etarios de los inmigrantes. A nuestro país llegan principalmente personas entre 20 y 50 años, lo que implica que ya hay un porcentaje de estos hombres, algunos de ellos de raza negra, que se encuentran en la edad en que se recomienda comenzar con los programas de detección precoz para cáncer de próstata (entre los 45 y 50 años) (Figura 3$)^{2}$. Si la permanencia de estos inmigrantes se prolonga, en 5 o 10 años más, constituirán una población significativa de hombres mayores de 40 años en los cuales, la raza negra es un factor de riesgo muy importante respecto de la historia natural de la enfermedad, como se discutirá más adelante. 

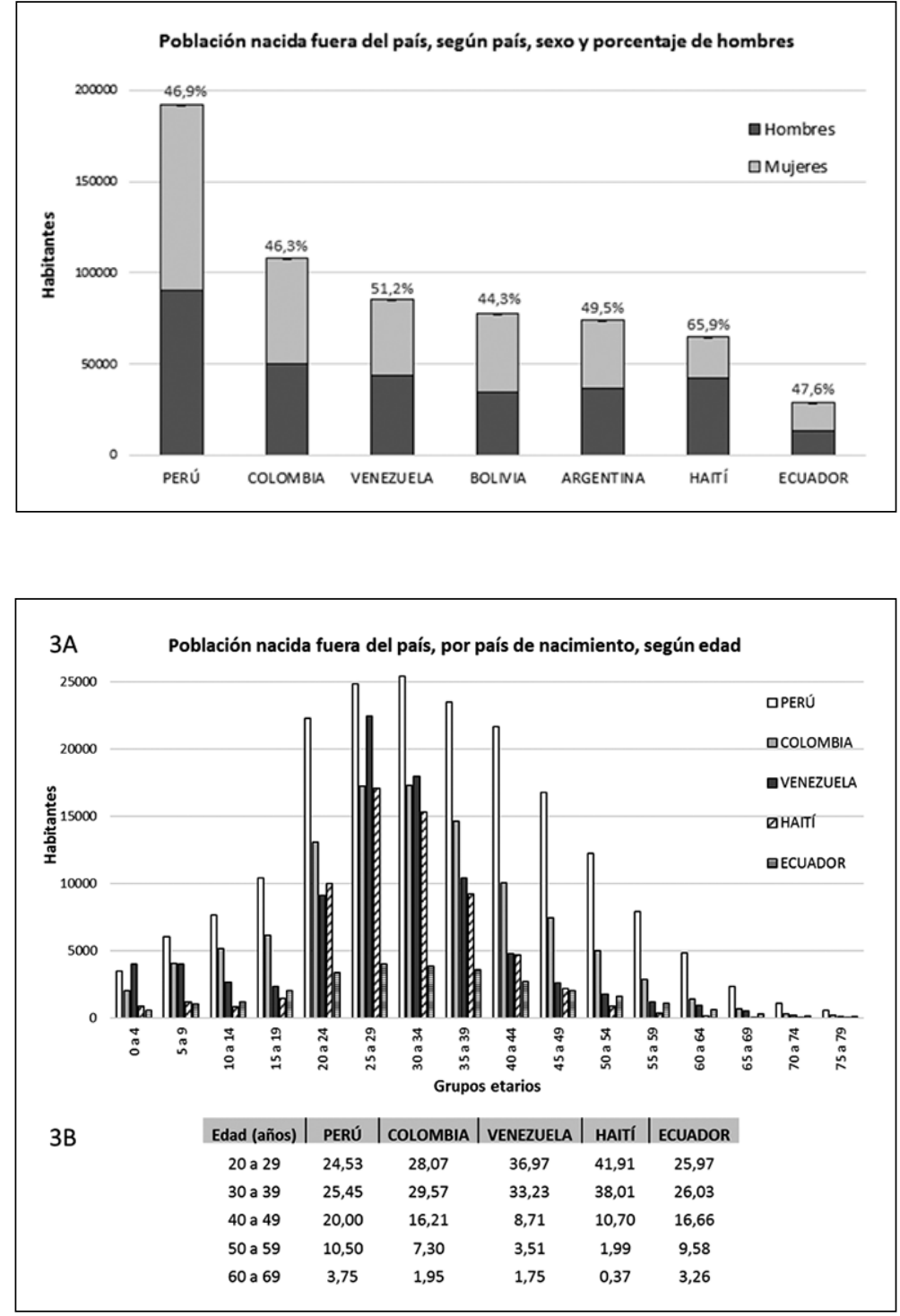

Figura 2. Población nacida fuera del país, según país, sexo y porcentaje de hombres. Fuente: Instituto Nacional de Estadísticas².
Figura 3. Población nacida fuera del país según edad y país de nacimiento. Fuente: Instituto Nacional de Estadísticas, Chile, $2018^{2}$.

\section{Epidemiología del cáncer de próstata en los países latinoamericanos}

A nivel mundial, las estimaciones de GLOBOCAN indican que el 2018 habrá cerca de 1,3 millones de casos nuevos y 359.000 muertes asociadas a cáncer de próstata. En cerca de la mitad de los países del mundo, es el cáncer más frecuentemente diagnosticado ${ }^{4}$.

En muchos países desarrollados hay una alta incidencia, que no se corresponde necesariamente a la mortalidad, lo que se explica por programas adecuados de detección precoz y acceso a terapias. La mayor mortalidad a nivel mundial se encuentra en los países del África sub-sahariana y el Caribe (Barbados, Jamaica y Haití), donde hay un predominio de hombres de raza negra ${ }^{4}$.

Las estimaciones de incidencia y mortalidad para Chile son mucho mayores a los otros países de Sudamérica (Tabla 2) ${ }^{4}$. Esto no se relaciona necesariamente con una mayor predisposición de la población chilena, ya que en nuestro país puede haber mayores tasas de detección o más reportes que mejoren las estadísticas. 
Tabla 2. Número de muertes y casos nuevos estimados de cáncer de próstata en hombres de todas las edades en países de Latinoamérica para el 2018

\begin{tabular}{|c|c|c|c|c|c|c|}
\hline \multirow[t]{2}{*}{ País } & \multicolumn{3}{|c|}{ n estimado de muertes } & \multicolumn{3}{|c|}{ n estimado de nuevos casos } \\
\hline & $\mathbf{n}$ & Tasa cruda* & TEE (mundo)* & $\mathbf{n}$ & Tasa cruda* & TEE (mundo)* \\
\hline Argentina & 3.974 & 18,2 & 12,3 & 11.600 & 53,0 & 42,4 \\
\hline Chile & 2.270 & 25,2 & 15,8 & 6.576 & 72,9 & 51,2 \\
\hline Colombia & 3.166 & 13,0 & 12,0 & 12.712 & 52,3 & 49,8 \\
\hline Ecuador & 1.341 & 15,9 & 13,9 & 3.322 & 39,4 & 38,8 \\
\hline Haití & 1.296 & 23,6 & 36,2 & 1.998 & 36,4 & 55,4 \\
\hline Perú & 2.721 & 16,7 & 15,6 & 7.598 & 46,7 & 47,8 \\
\hline Venezuela & 3.399 & 21,1 & 23,3 & 7.445 & 46,2 & 51,2 \\
\hline
\end{tabular}

TEE: Tasa estandarizada por edad. *tasa por 100.000 habitantes. Fuente: Globocan $2018^{4}$.

Tabla 3. Tasas de incidencia y mortalidad (por 100.000 habitantes) por cáncer de próstata

\begin{tabular}{|lccccc|}
\hline Cáncer de próstata & $\begin{array}{c}\text { Todas las } \\
\text { razas }\end{array}$ & $\begin{array}{c}\text { Blanco no } \\
\text { hispano }\end{array}$ & $\begin{array}{c}\text { Negro no } \\
\text { hispano }\end{array}$ & $\begin{array}{c}\text { Asiático/lslas } \\
\text { del Pacífico }\end{array}$ & $\begin{array}{c}\text { Hispano/ } \\
\text { Latino }\end{array}$ \\
\hline Incidencia, 2010-2014 & 114,9 & 107,0 & 186,8 & 58,4 & 97,0 \\
\hline Mortalidad, 2011-2015 & 19,5 & 18,2 & 40,8 & 8,7 & 16,1 \\
\hline
\end{tabular}

Fuente: Incidencia, North American Association of Central Cancer Registries, 2017; Mortalidad- National Center for Health Statistics, Centers for Disease Control and Prevention, $2017^{5}$

El cambio de país puede provocar un cambio en las tasas de incidencia y mortalidad de la población migrante, ya sea por un mayor riesgo de presentar o más probabilidad de detectar la enfermedad. Chile presenta mayor control de enfermedades infecciosas y nutricionales respecto de otros países latinoamericanos, lo que puede aumentar la expectativa de vida y la posibilidad de manifestar enfermedades no infecciosas, lo que, sumado a mejores estrategias de screening, podrían cambiar los indicadores poblacionales de cáncer de próstata.

\section{El cáncer de próstata en hombres de raza negra}

A pesar del escaso conocimiento de las causas del cáncer de próstata, se ha observado que el componente racial está relacionado con el riesgo de tener cáncer y de su agresividad (Tabla 3$)^{5}$. La mayor incidencia se observa en hombres de raza negra, lo que ha sido ampliamente descrito en Estados Unidos y el Caribe, reflejando una pre- disposición étnica ${ }^{4}$. Sin embargo, algunos estudios sobre cáncer de próstata en afroamericanos en Estados Unidos, han propuesto que adicionalmente, hay menor acceso a la salud en este grupo; incluso se ha propuesto la oportunidad y calidad de la cirugía es de menor calidad respecto de otros grupos raciales 6 .

En Estados Unidos, los hombres de raza negra tienen $74 \%$ más de riesgo de tener cáncer de próstata respecto de la población blanca. Por lo tanto, la ascendencia debe ser considerada al momento de establecer el riesgo, junto a la edad, la historia familiar y algunas condiciones genéticas. Al comparar los índices (Tabla 3), resulta evidente concluir que el aumento de hombres de raza negra en nuestro país, al ser atendidos en nuestro sistema de Salud, podría modificar la incidencia y mortalidad por cáncer de próstata si no se toman medidas apropiadas.

Un estudio reciente de screening con Antígeno Prostático Específico (APE) entre hombres de distintas razas, indicó que, al comparar el riesgo de desarrollar enfermedad preclínica, los hombres negros presentan $28 \%$ a $56 \%$ más de riesgo res- 
pecto de la población general, presentando, 33 a $70 \%$ más de riesgo relativo de diagnóstico clínico. Entre hombres que desarrollan la enfermedad clínica, el riesgo de diagnosticarlos usando APE, es similar para todas las razas. Además, entre los pacientes con enfermedad preclínica, los hombres negros tienen 44 a $75 \%$ más de riesgo de metástasis antes del diagnóstico, lo que indica mayor riesgo de progresión de esta población. Las diferencias en la incidencia entre ambas poblaciones están dadas por el desarrollo de cáncer de próstata preclínico, por lo que las estrategias de screening deberían ser distintas. Los hombres negros tienen mayor riesgo de desarrollar cáncer a temprana edad y de progresar a cáncer metastásico o de alto grado antes del diagnóstico clínico, por lo que es fundamental el screening a menor edad o con mayor intensidad que el resto de la población ${ }^{6}$.

\section{Susceptibilidad genética}

Hasta el momento no se conocen con certeza las causas de la diferencia en susceptibilidad y agresividad del cáncer de próstata en hombres de distintas razas, aunque parte de ellas podrían estar dadas por alteraciones genéticas y moleculares. El inicio de la carcinogénesis prostática, en la gran mayoría de los casos, se debería a la interacción entre múltiples alelos comunes con penetrancia intermedia y factores ambientales, como la infección e inflamación, lo que explica la baja prevalencia de cáncer hereditario $(<5 \%)$. El cáncer familiar es más frecuente, cuyo riesgo está determinado por la raza, ya que tener 2 o más parientes afectados en primer grado se asocia con un riesgo relativo de 9,7 en afrodescendientes, comparados con 3,9 en caucásicos. Las alteraciones genéticas observadas en los tumores son distintas, relacionándose con la mayor agresividad tumoral. En tumores de afrodescendientes hay mayor frecuencia de pérdida de copias de los genes MSH2 y MSH6 (reparadores de DNA) ${ }^{7}$, de mutaciones en la región de susceptibilidad 8q24 (donde hay una serie de genes y RNAs no codificantes $)^{8}$ y de mutaciones oncogénicas "driver", siendo $30 \%$ de ellas novedosas para cáncer de próstata. Además, no se observa fusión del gen ERG ni mutaciones en PIK3C, hay menos pérdida de PTEN y ganancia en CCND1 y MYC, y cambios en la expresión de genes relacionados con la regulación del Calcio?. Aún se desconocen las causas de estas mutaciones y carcinógenos específicos, por lo que la predis- posición a tener cáncer de próstata podría ser una combinación de factores ambientales y genéticos ${ }^{7}$.

\section{Riesgo de cáncer de próstata en Latinoamérica}

En Latinoamérica existen pocos antecedentes sobre el riesgo de desarrollar cáncer de próstata en la población afrodescendiente. En Colombia se observó que a pesar de la disminución de la mortalidad a nivel nacional, esta es mayor al promedio en la población afrodescendiente ${ }^{10}$. Un estudio realizado para comparar país de origen y raza en países caribeños reportó que los hombres de raza negra presentaron una tasa de mortalidad de 35,5 por 100.000 habitantes, muy superior a la tasa de 19,1 observada en hombres de todas las razas ${ }^{11}$.

El aumento de los hombres afrodescendientes en nuestro país, inevitablemente debe cambiar el paradigma de screening y tratamiento del cáncer de próstata. En un momento donde las migraciones y mezcla de diferentes culturas es cada vez más frecuente, es necesario considerar su efecto en la salud pública.

\section{Efecto de la inmigración en la salud del país receptor}

La migración provoca cambios demográficos que modifican los índices de la población nativa. Cambios en la tasa de mortalidad, natalidad y esperanza de vida son habituales en estos procesos. Estimando que estas migraciones se relacionan prioritariamente con una mejor situación laboral, migran habitualmente más hombres que mujeres. Esto genera un desequilibrio en el volumen por sexo y un mayor impacto en el género masculino ${ }^{12}$. Este efecto toma especial interés cuando se estudia el impacto en la salud debido a esta interacción, generando dos posibles escenarios: que la población migrante genere un gran impacto en la salud modificando los indicadores de salud local o que la población migrante se establezca y a largo plazo tienda a no afectar al país receptor. Variantes de estas dos realidades surgen a diario en el intercambio cultural de la globalización. El resultado dependerá de la cantidad de población migrante, la eficiencia de los cambios para prevalecer en las siguientes generaciones, entre otros factores. Independiente del resultado, esta interacción debe ser analizada con el fin de aportar la mayor cantidad de antecedentes que permitan fortalecer el sistema de salud. 
Tabla 4. Incidencia de pobreza multidimensional por lugar de nacimiento, 2015-2017

\begin{tabular}{|lccc|}
\hline $\begin{array}{l}\text { Incidencia pobreza } \\
\text { nacimientos 2017 }\end{array}$ & $\begin{array}{c}\text { Estimación \% lugar de } \\
\text { nacimiento }\end{array}$ & Error estándar 2017 & $\mathbf{N}^{\circ}$ personas 2017 \\
\hline Nacidos fuera de Chile & 24,6 & 2,5 & 178.794 \\
\hline Nacidos en Chile & 20,5 & 0,3 & 3.312 .928 \\
\hline Promedio país & 20,7 & 0,4 & 3.530 .889 \\
\hline
\end{tabular}

Fuente: Ministerio de Desarrollo Social, Encuesta Casen $2017^{11}$.

El impacto económico de la salud pública debido a la migración es considerable y puede generar una disminución de la calidad y frecuencia de las prestaciones de salud pública. Actualmente, el programa de salud 2018-2022, contempla cambios y modernización del sistema de salud público, pero no contempla un plan especial para abordar los cambios que podría ocasionar la población inmigrante en Chile $^{13}$. La creación de servicios de salud inclusivos y universales debería ser un foco prioritario a la hora de mejorar la calidad de atención al paciente. Todo programa de salud o política pública debe ser accesible e inclusivo, sin importar condición migratoria, nacionalidad, etnia o religión ${ }^{14}$.

Por esto, es importante considerar las condiciones en las que viven los migrantes en Chile. Según la encuesta Casen $2017^{15}$, aproximadamente 10,8\% (cerca de 200.000 personas) viven en pobreza o pobreza extrema, situación que dificulta el acceso a servicios y mejores condiciones de salud (Tabla 4). El proceso migratorio puede exponer a mayores riesgos y al aumento de la vulnerabilidad biológica, social, psicológica, económica y cultural, por lo que deben ser analizadas en forma regular, con la finalidad de prevenir y proteger las condiciones de vida de migrantes y las posibles consecuencias en su salud. Cuando una migración se realiza en forma ordenada, cumpliendo los requerimientos básicos de cuidado y la adecuada regularización del estatus migratorio, puede resultar en una mejora de las condiciones socio ambientales, mayor acceso a servicios y mejores condiciones de salud para la población migrante ${ }^{14}$.

Es importante tener presente que, al cambiar de país, se puede modificar el riesgo de sufrir una enfermedad. Cambios en los hábitos alimenticios, a la exposición de agentes patógenos o mayor acceso a los sistemas preventivos de Salud, pueden hacer que, en nuestro país, los migrantes tengan menor riesgo de sufrir algunas enfermedades y aumentar su riesgo de morir por cáncer de próstata. Sólo como un antecedente, la esperanza de vida en Chile es la mayor comparada con otros países latinoamericanos (Tabla 5) ${ }^{16}$. Dado que la edad es uno de los principales factores de riesgo para desarrollar cáncer de próstata ${ }^{17}$, al aumentar la esperanza de vida de los inmigrantes en nuestro país, aumentaría en ellos el riesgo de desarrollar este cáncer. Esto además podría acrecentar la leve tendencia al alza que presenta actualmente la incidencia nacional de cáncer de próstata.

El migrante afrodescendiente que llega a nuestro país se caracteriza por su juventud y buena salud, pero debe enfrentar barreras socioculturales, afectando directamente el bienestar psicosocial del individuo. La historia de esclavitud y discriminación sistemática de los afrodescendientes condujo a un aumento de cargas sociales, económicas y de salud que aún perduran en la actualidad.

Frente al escenario migratorio actual en Chile, uno de los pilares fundamentales es la educación de los nuevos pacientes. El cáncer de próstata es

Tabla 5. Esperanza de vida al nacer (en años) en los países Latinoamericanos en el año 2016 según la Organización Mundial de la Salud ${ }^{22}$

\begin{tabular}{|lcc|}
\hline País & \multicolumn{2}{c|}{ Años } \\
\hline Haití & 63,5 & 61,3 \\
\hline Bolivia & 71,5 & 69,1 \\
\hline Venezuela & 74,1 & 69,5 \\
\hline Colombia & 75,1 & 71,5 \\
\hline Perú & 75,9 & 73,4 \\
\hline Ecuador & 76,5 & 74,1 \\
\hline Argentina & 76,9 & 73,5 \\
\hline Chile & 79,5 & 76,5 \\
\hline
\end{tabular}


una patología GES lo que permite a los pacientes acceder a un tratamiento de calidad independiente de sus recursos económicos, los que en la mayoría estos pacientes son de recursos limitados. Con el fin de favorecer la optimización de los recursos y el acceso oportuno a la salud, en particular en este caso, detectar el cáncer cuando se encuentra en una fase localizada, factible de curar, es fundamental considerar si es necesario que se tome medidas preventivas especiales con estos pacientes.

\section{Medidas sugeridas para el screening en hom- bres de raza negra}

Existe una controversia en la actualidad respecto al beneficio del screening en la población general para evitar muertes por cáncer de próstata ${ }^{9}$. Específicamente, la evidencia recolectada hasta el momento tampoco indica que el screening rutinario con APE en hombres de raza negra pueda prevenir muertes por cáncer de próstata ${ }^{18}$.

Si bien las principales guías clínicas urológicas (National Comprehensive Cancer Network [NCCN]; American Urological Association [AUA]; European Association of Urology [EAU]), coinciden en reconocer categóricamente a los hombres de raza negra como un grupo de riesgo tanto para el desarrollo como progresión del cáncer de próstata, no son categóricas al momento de hacer recomendaciones específicas sobre screening en esta población: la NCCN considera "razonable" (sic) que los afroamericanos inicien la discusión sobre screening con APE "varios" (sic) años antes que los caucásico-americanos y considerar el screening anual más que el de año por medio; la AUA reconoce que el screening en hombres menores a 55 años podría tener algún beneficio en los grupos de mayor riesgo (incluye a los hombres de raza negra), siempre de manera informada e individualizada, sin dar patrones explícitos sobre cómo y desde qué edad hacerlo; la misma AUA no hace diferencias según raza en sus recomendaciones de screening para hombres entre 55 y 69 años; la EAU propone ofrecerlo a hombres de raza negra desde los 45 años, siempre que estén bien informados respecto a los beneficios y riesgos ${ }^{5,6,18}$. Mientras que en Chile, las guías clínicas del MINSAL no incluyen recomendaciones para los hombres de raza negra ${ }^{19}$.

Un aspecto adicional a considerar, son los umbrales de APE para predecir riesgo de cáncer prostático. En 1996, Morgan et al. reportaron que el nivel de APE en hombres de raza negra es significativamente mayor que en blancos, de modo que, si se utilizan los rangos tradicionales de referencia según edad, $41 \%$ de los casos de cáncer prostático no serían diagnosticados ${ }^{20}$. El mismo estudio propuso que para que el APE mantenga 95\% de sensibilidad en hombres de raza negra, se deberían usar los siguientes rangos: 40-49 años, 0 a $2,0 \mathrm{ng} / \mathrm{mL}$; $50-59$ años, 0 a $4,0 \mathrm{ng} / \mathrm{mL} ; 60-69$ años, 0 a 4,5 ng/mL; 70-79 años, 0 a 5,5 ng/mL. Un estudio más reciente, reportó que el valor óptimo para predecir riesgo de cáncer prostático a 4 años de seguimiento en hombres mayores de 40 años fue de $2,5 \mathrm{ng} / \mathrm{mL}$ en caucásicos y $1,9 \mathrm{ng} /$ $\mathrm{mL}$ en afroamericanos ${ }^{21}$. Sin embargo, aún no hay un consenso respecto a los valores umbrales a utilizar específicamente en esta población con fines de screening.

\section{Conclusiones}

Los eventos migratorios son cada vez más frecuentes a nivel mundial. Estos repercuten en distinta magnitud en la salud de los migrantes y en los sistemas de salud de los países receptores. Nuestro país vive un fenómeno singular en este sentido, ya que, debido a la migración, ha aumentado el número de hombres de raza negra entre 20 y 50 años. Esta población presenta mayor riesgo de desarrollar cáncer de próstata, de manera más temprana y agresiva respecto de la población caucásica, principal fenotipo de la población chilena.

El enfoque actual de las políticas migratorias debería incluir algunas políticas específicas respecto de riesgos específicos de esta población, estableciendo recomendaciones o guías de manejo y educación sobre el diagnóstico precoz enfocadas en esta población de alto riesgo, para lo que, además, inicialmente debería ser cuantificada y evaluada en su distribución a nivel nacional.

Finalmente, hay que considerar que un aumento en la prevalencia de cáncer de próstata repercutirá de alguna manera en la economía nacional considerando que es una patología GES y gran parte de los inmigrantes son de escasos recursos. Es imperante establecer el efecto $\mathrm{y}$ tomar las medidas pertinentes para afrontar este cambio. 


\section{Referencias}

1. Rojas Pedemonte N, Koechlin J. Migración haitiana hacia el sur andino. 1st ed. Universidad Antonio Ruiz de Montoya, Breña, Perú, 2017.

2. Instituto Nacional de Estadísticas: Segunda entrega definitiva resultados censo 2017. Santiago, 2018.

3. Rojas Pedemonte N, Silva Dittborn C, Amode N, Vásquez J, Orrego C. Migración haitiana en Chile (Boletín DEM N ${ }^{\circ}$ 1). Santiago, 2016.

4. Bray F, Ferlay J, Soerjomataram I, Siegel RL, Torre LA, Jemal A. Global cancer statistics 2018: GLOBOCAN estimates of incidence and mortality worldwide for 36 cancers in 185 countries. CA Cancer J Clin, 2018.

5. American Cancer Society. American Cancer Society. Cancer Facts \& Figures 2018.

6. Tsodikov A, Gulati R, de Carvalho TM, Heijnsdijk EAM, Hunter-Merrill RA, Mariotto AB, et al. Is prostate cancer different in black men? Answers from 3 natural history models. Cancer 2017; 123: 2312-9.

7. Tan S-H, Petrovics G, Srivastava S. Prostate Cancer Genomics: Recent Advances and the Prevailing Underrepresentation from Racial and Ethnic Minorities. Int J Mol Sci 2018; 19: 1255.

8. Han Y, Rand KA, Hazelett DJ, Ingles SA, Kittles RA, Strom SS, et al. Prostate Cancer Susceptibility in Men of African Ancestry at 8q24. J Natl Cancer Inst 2016; 108 (7).

9. Jaratlerdsiri W, Chan EKF, Gong T, Petersen DC, Kalsbeek AMF, Venter PA, et al. Whole-Genome Sequencing Reveals Elevated Tumor Mutational Burden and Initiating Driver Mutations in African Men with Treatment-Naïve, High-Risk Prostate Cancer. Cancer Res 2018; 78: 6736-46.

10. Matiz JLP, Reyes NJA, Becerra MPS, Almendrales FPD. Evolución de la mortalidad por cáncer de próstata en
Colombia: estudio ecológico. Urol Colomb 2014; 23 : 3-10.

11. Pinheiro PS, Callahan KE, Ragin C, Hage RW, Hylton T, Kobetz EN. Black Heterogeneity in Cancer Mortality: US-Blacks, Haitians, and Jamaicans. Cancer Control 2016; 23: 347-58.

12. Índices de intensidad migratoria México-Estados Unidos 2010. México, D.F., 2012.

13. Piñera S. Programa de Salud 2018-2022. 2018.

14. Cabieses B, Bernales M, McIntyre A. La migración internacional como determinante social de la salud en Chile: evidencia y propuestas para políticas públicas. 1st ed. Universidad del Desarrollo, 2017.

15. Observatorio Social: Situación de pobreza, Síntesis de Resultados Casen 2017. 2018.

16. World Health Organization. Global Health Observatory (GHO) data: Life expectancy. 2018.

17. Cuzick J, Thorat MA, Andriole G, Brawley OW, Brown $\mathrm{PH}$, Culig Z, et al. Prevention and early detection of prostate cancer. Lancet Oncol 2014; 15: e484-92.

18. Cooperberg MR. The New US Preventive Services Task Force "C" Draft Recommendation for Prostate Cancer Screening. Eur Urol 2017; 72: 326-8.

19. Ministerio de Salud Gobierno de Chile: Guía clínica AUGE Cáncer de próstata en personas de 15 años y más. 2015.

20. Morgan TO, Jacobsen SJ, McCarthy WF, Jacobson DJ, McLeod DG, Moul JW. Age-Specific Reference Ranges for Serum Prostate-Specific Antigen in Black Men. N Engl J Med 1996; 335: 304-10.

21. Sutton SS, Crawford ED, Moul JW, Hardin JW, Kruep E. Determining optimal prostate-specific antigen thresholds to identify an increased 4-year risk of prostate cancer development: an analysis within the Veterans Affairs Health Care System. World J Urol 2016; 34: 1107-13. 